\title{
Ambiguity about the volume of colloids administered in a clinical study of thoracic esophagectomy precludes conclusions on the renal safety of hydroxyethyl starch
}

\author{
Christian J. Wiedermann ${ }^{1}$ D \\ Received: 22 January 2019 / Accepted: 7 March 2019 / Published online: 18 March 2019 \\ (c) Springer Nature Singapore Pte Ltd. 2019
}

Keywords Esophageal cancers · Esophagectomy $\cdot$ Hydroxyethyl starch 6\% 130/0.4/9

Dear Editor,

In a recent edition of Surgery Today, Fujita et al. [1] reported that hydroxyethyl starch 6\% 130/0.4/9 (HES) was well tolerated in esophagectomy with 3-field lymph-node dissection. Acetate Ringer's solution was administered during esophagectomy, together with either human serum albumin (HSA) or HES. In the "Methods" section of the publication, it is stated that "a total of $100 \mathrm{~mL}$ of either HSA solution or HES 130/0.4/0 (Voluven $\left.{ }^{\circledR}\right)$ solution was administered during the thoracic procedure $(. .$.$) at 150-250 \mathrm{~mL} / \mathrm{h}$, dependent on the patient's status and the duration of the thoracic procedure". Since the total time of the thoracic procedure was $190.0 \mathrm{~min}$ in the HSA group and $177.2 \mathrm{~min}$ in the HES group, the statement that $100 \mathrm{~mL}$ was given as the total volume may be an error. If it is correct that the total volume given was $100 \mathrm{~mL}$, then the mean duration of the infusion would have been only around $30 \mathrm{~min}$, which we do not expect would have had hemodynamic efficacy at the given infusion rate. In that case, any conclusions about HES safety are irrelevant, as the usual minimum volume of
HES solution administered to adults is $500 \mathrm{~mL}$. In order to draw meaningful conclusions from the study, readers need to know the true amounts of colloids that were administered.

\section{Compliance with ethical standards}

Conflict of interest CJW received lecture fees from and served as a consultant to CSL Behring (Germany) and Grifols (Spain).

\section{Reference}

1. Fujita T, Okada N, Horikiri Y, Sato T, Fujiwara H, Mayanagi S, et al. Safety and efficacy of hydroxyethyl starch 6\% 130/0.4/9 solution versus $5 \%$ human serum albumin in thoracic esophagectomy with 3-field lymph nodes dissection. Surg Today. 2019. https:// doi.org/10.1007/s00595-018-1752-6.

Publisher's Note Springer Nature remains neutral with regard to jurisdictional claims in published maps and institutional affiliations.
Christian J. Wiedermann

christian.wiedermann@ext.umit.at

1 UMIT-University of Health Sciences, Medical Informatics and Technology, Eduard-Wallnöfer-Platz 1, 6060 Hall in Tyrol, Austria 